\title{
CC Carriage Lifting Extension Analysis
}

\author{
Engineering Note \#3740.210-EN-85
}

\author{
Revised August 7, 1987
}

Revised by: Richard D. Luther 
Pinned Extensions for eC Correrisere

ex $6 / 25 \% 7$

Extensions cull be pinned to the support brackets in order to prow de pickup points for lifting the Ge calorimeter facrrige combination. The extension must be long enough to ensure that the lifting cables will not bear on the cryostat heads. The to tail weight of the ce from Multiplan (Rev N) is:

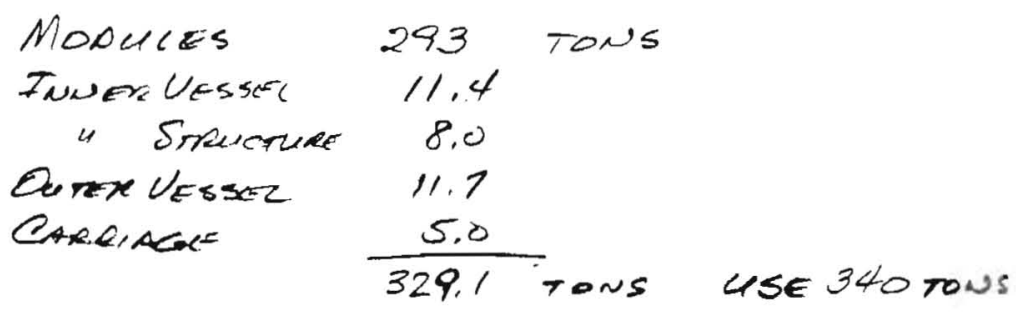

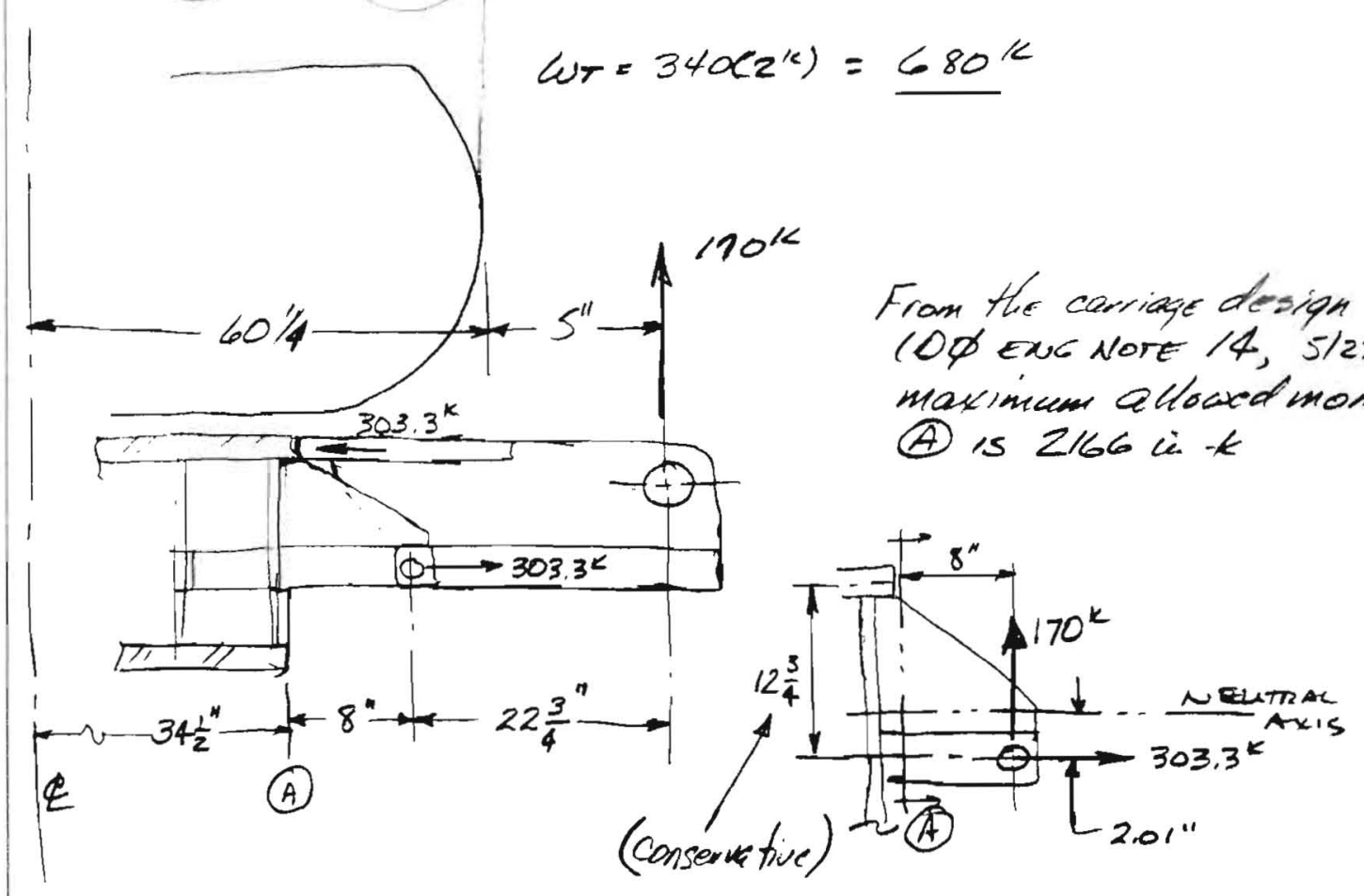

$$
\begin{aligned}
M_{0} & =170(8)+303.3(2.01) \\
& =1360+609.6 \\
& =1970 \mathrm{im} \cdot \mathrm{k}
\end{aligned}
$$

Impact FActors $\left(\frac{2166}{1970}\right)=10 \%$ 
$\sec 6 / 20 / 87=$

Checic Csavar TutenFaces

Bexperne@TOA DeAte

$106 \frac{1}{2} R$

$21 \frac{7}{8}$

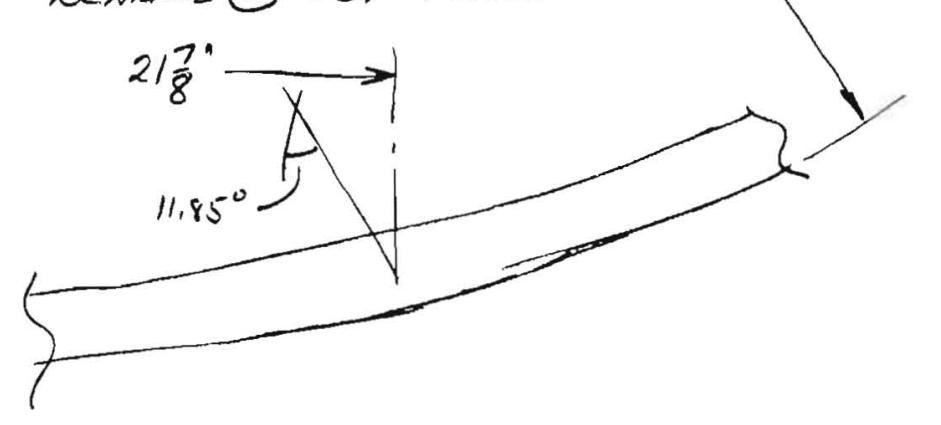

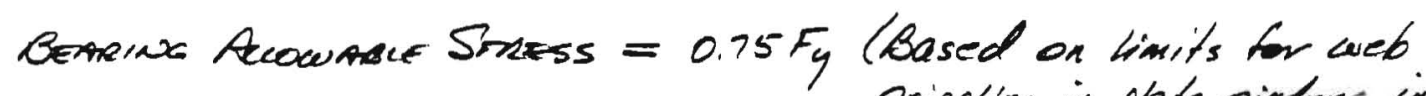
$=0,75(30)$ Crippling in plate qinders in $=0,75(30)$ AIse specification for Bidss)
$=22,5 \mathrm{ks}$;

$\frac{\rho}{A}<22.5$

$A>\frac{303.3}{22.5}$

$A>13.5$ in $^{2}$

for $1 \frac{1}{2}$ " leate

$A=1 \frac{1}{2} \times C$

$L=\frac{13.5}{1.5}=9^{11}$

$\therefore$ Use 10 "aipe top peate

on ex reusion. Throkuess (say 2")

AnS AnteE TO BE

sufficient to coner

ruce pharavess op apdedse PLATE:

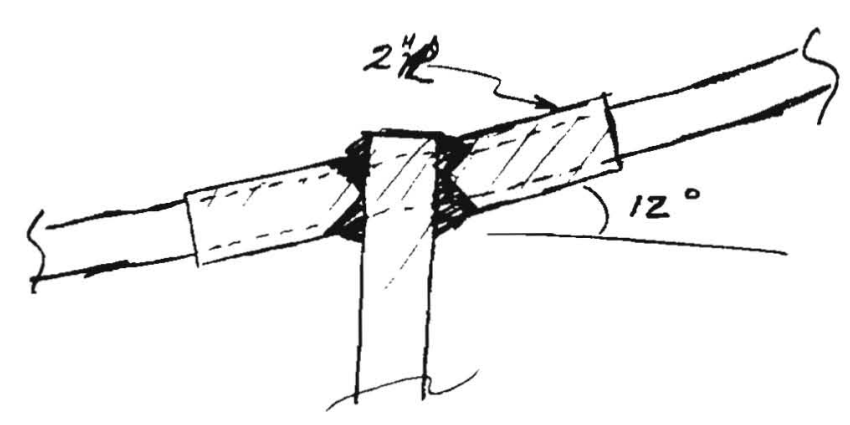




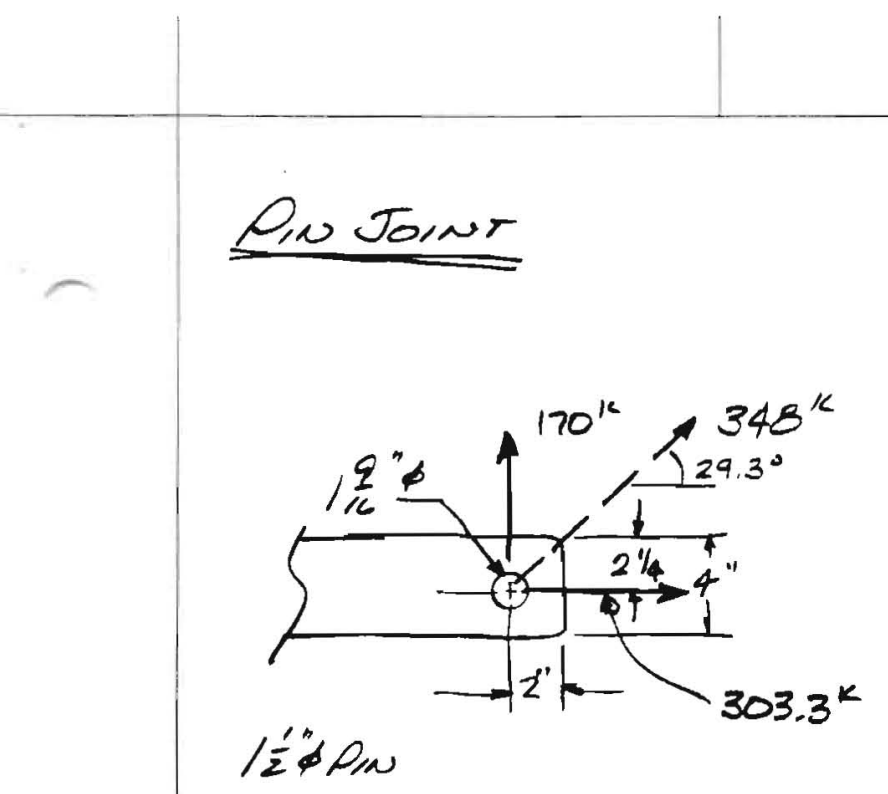

BeAsing

$$
\begin{aligned}
& F_{p}=0.9 F_{4} \quad \text { (AISC 15.1.5.1) } \\
& =0.9(30)=27 \mathrm{ks} \text {, } \\
& f_{p}=\frac{\rho}{A}=\frac{348}{1,5(10 \%)}=22,6 \mathrm{ks} /<27
\end{aligned}
$$

SHEAR OuT

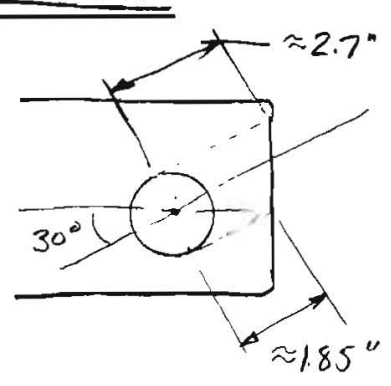

$F_{5}=0.4 F_{4}$ CAISC

$$
\begin{aligned}
& =0.4(30) \\
& =12 \mathrm{ks}
\end{aligned}
$$

$$
f_{s}=\frac{348^{\mathrm{k}}}{(1,85+2.7)^{\left.10 \frac{1}{4}\right)}}=7,46 \mathrm{ks}
$$

Hon,zoutte

$$
f_{s}=\frac{303.3}{2(2)(10 \%)}=7.40 \mathrm{ks} /
$$

Pin

Shear: $F_{\nu}=0.22 F_{u}$

$$
\tau=\frac{P}{4}=\frac{348}{\frac{\pi(1,5)^{2} \times x}{4}} ; x=\begin{gathered}
\text { number } \\
\text { of shear } \\
\text { planes. }
\end{gathered}
$$$$
x=6
$$

$$
\begin{aligned}
& \tau=\frac{348}{1.7 \omega 1(6)}=32.8<F_{\nu} \\
& 0.22 \mathrm{Fu}_{u}>32.8 \\
& F_{u}=150 \mathrm{ks}, \quad 7001+16 \mathrm{H} .
\end{aligned}
$$$$
\underline{x=8}
$$

$$
\begin{gathered}
r=\frac{348}{1.768(8)}=24.6 \mathrm{ks} ; \\
0.22 F_{4}>24.6 \\
F_{4}>112 \mathrm{ks}
\end{gathered}
$$

$\therefore$ USE MATERIAL MAUING TEUSILE STEEUGTH in excess of $1 / 2 \mathrm{ks}$.

Surae

$$
\begin{aligned}
& z=\frac{P}{A}=0.4 F_{Y}=12 \mathrm{ks} \\
& z=\frac{170}{4\left(10^{1 / 4}\right)}=4.15 \mathrm{ks}, 0 / 2
\end{aligned}
$$

Tension

$$
\begin{aligned}
& f_{a}=\frac{\rho}{A}<F_{a}=0.6 F_{9}=18 \mathrm{ks}, \\
& f_{a}=\frac{303.3}{\left(4-\frac{P}{10}\right)(10 \%)}=12.1 \mathrm{ks} \text { O.l }
\end{aligned}
$$




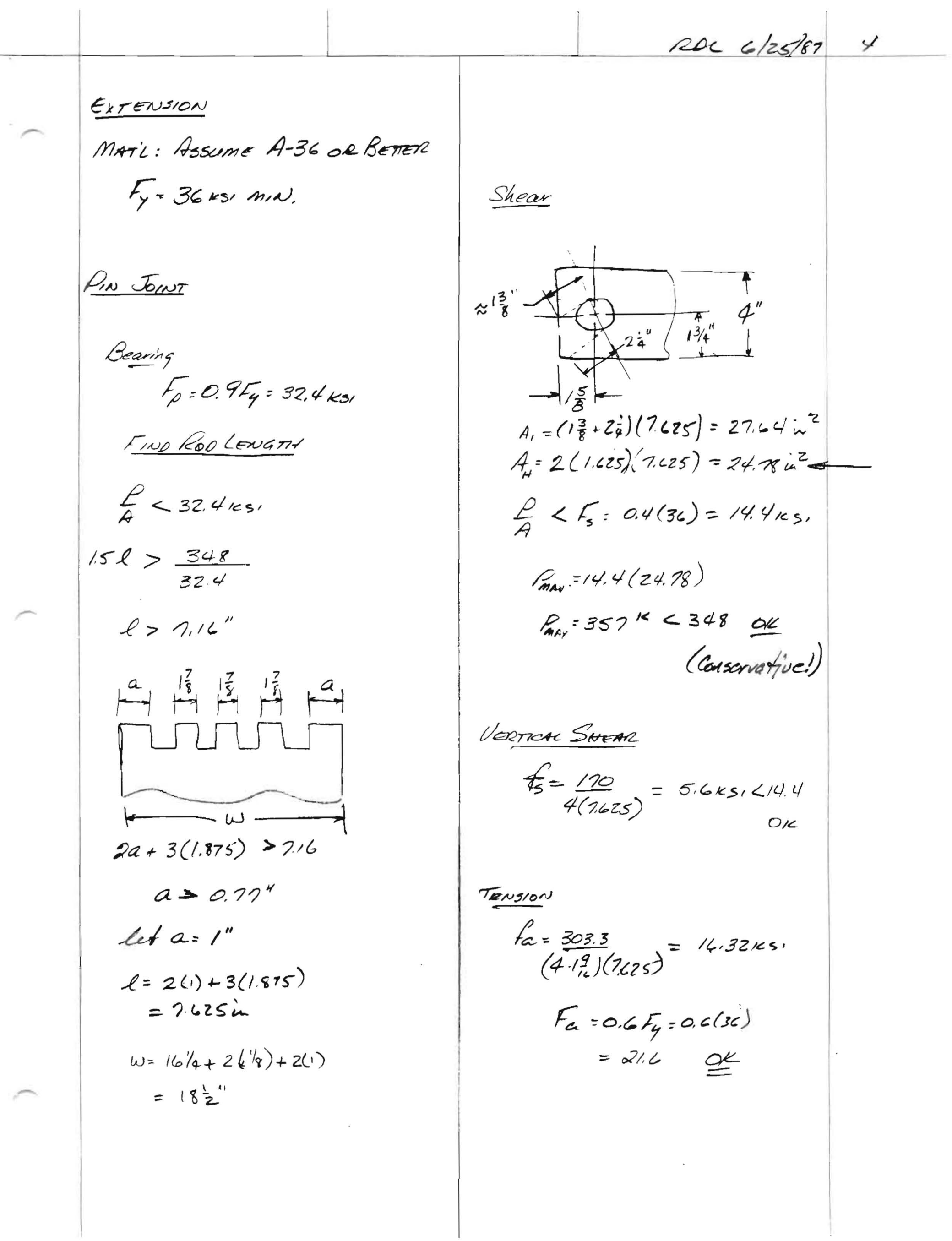




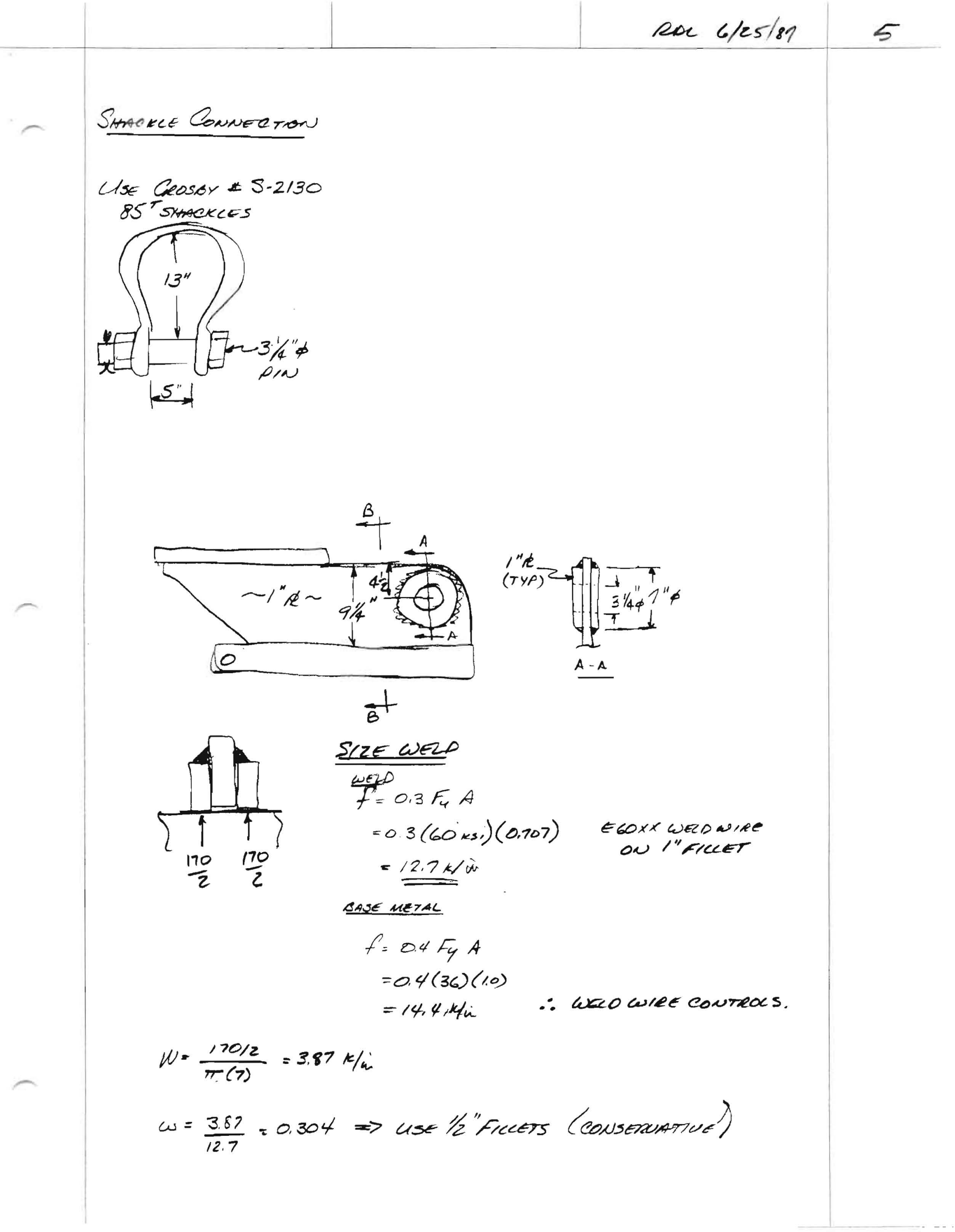




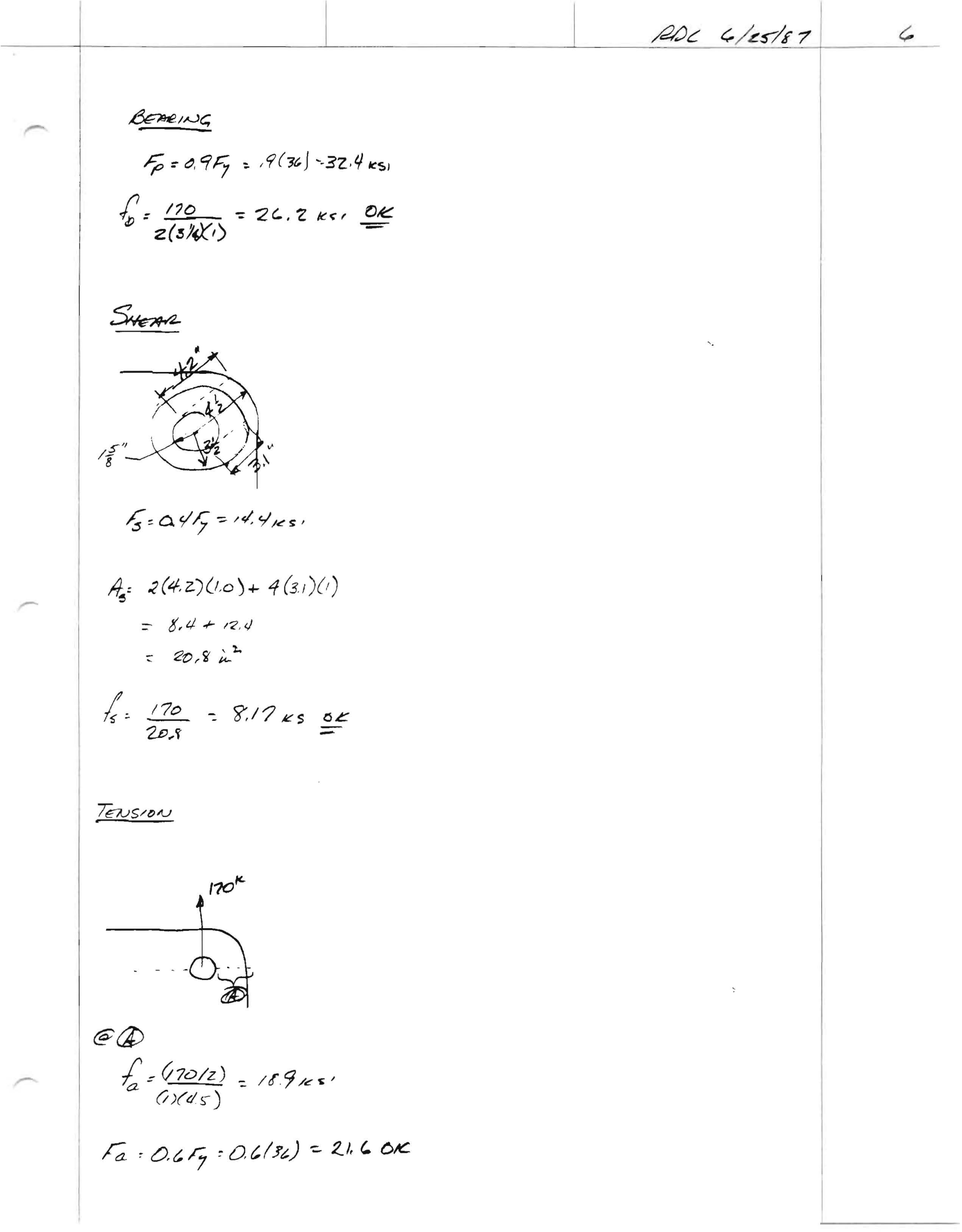


$120 \times 6 / 26 / 87$

8

Chectwebshear@hole.

$$
\begin{aligned}
A_{w} & =1 \times(9-3+4) \\
& =9.75 \\
f_{s} & =\frac{170}{9.15}=17.4 \mathrm{ks}, \\
F_{5} & =0.4 F_{4}=14.4 .
\end{aligned}
$$

Considure Reinfacermat Paos

$$
\begin{aligned}
A_{w} & =9.75+2\left(1 \times 7-3 \frac{1}{4}\right) \\
& =9.75+7.5 \\
& =17.25 \\
f_{s} & =\frac{170}{17.25}=9.86<14.4 \text { ote }
\end{aligned}
$$

Check wet aaxy from hole

$$
\begin{aligned}
& A_{w}=1(9+4)=13 \\
& f_{s}=\frac{170}{13}=13.1 \mathrm{ks}<14.4 \mathrm{ok}
\end{aligned}
$$

Benbing - Compression Cou trots

AISC $1,5,114,5,2$

$$
\begin{aligned}
& l=223 / 4+8=303 / 4 \\
& r_{T}=\sqrt{\frac{(1 / 2)(3)(1)^{3}}{(1)(3)}}=0.289^{\prime \prime} \\
& l / r_{T}=106.5 \\
& C_{b}=1.0 \\
& \sqrt{\frac{\left.\left(510 \times 10^{3}\right) 1.0\right)}{F_{4}}}=119>\mathrm{l} / r_{T}
\end{aligned}
$$

$$
\begin{aligned}
F_{b} & =\left[\frac{2}{3}-\frac{F_{y}\left(l e / r_{r}\right)^{2}}{1530 \times 10^{3} C_{b}}\right] F_{y} \\
& =\left[\frac{2}{3}-\frac{3\left((106.5)^{2}\right.}{1.53 \times 10^{6}}\right] F_{y} \\
& =0.4 F_{4} \\
& =0.4(36) \\
& =14.4 \mathrm{ks})
\end{aligned}
$$

$$
\begin{aligned}
& F_{b}=\frac{M_{\text {max }}}{M_{\text {MAx }}}=14.4(10.11) \\
& =146 \mathrm{in}-\mathrm{k}
\end{aligned}
$$

OBVIOUSLY CWACCETHALE, THEREROR, TOP FLANGE MUST Be considereco. Quteile SECTION B.B FOR YIELOMG:

$$
\begin{gathered}
F_{B}=0.6 F_{y} \\
0.6(36)=\frac{M_{\text {MAx }}}{10.11} \\
M_{\text {mAx }}=218.4 \mathrm{mi} \cdot \mathrm{k} \\
l=\frac{218.4}{170}=1.28^{\prime \prime}
\end{gathered}
$$

Wote that considser tion of full web heigkt ( $\left(9_{2}^{\prime} "\right)$ and reintoraing pals arll reduce this stress. Briag top tlange to within $13 / 4$ " of centerline of lale.

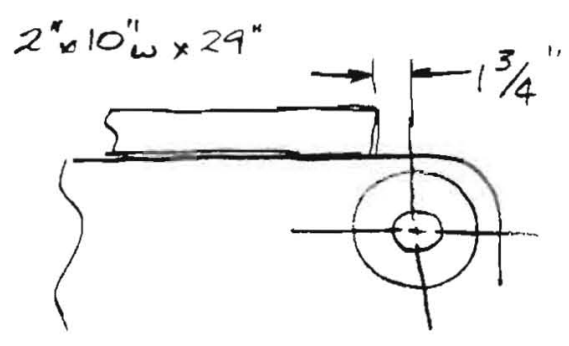


abe 6/26/87 9

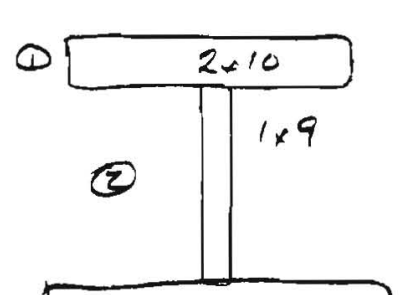

(3) $4 \times 14 \frac{1}{4}$

$$
\begin{aligned}
& \begin{array}{lccccc}
\text { (1) } & \frac{A}{20} & \frac{y}{14} & \frac{A 4}{280} & \frac{A d^{2}}{1455} & \frac{I_{0}}{C .7} \\
\text { (2) } & 9 & 8 \frac{1}{2} & 76.5 & 83 & 61 \\
\text { (3) } & \frac{52}{86} & 2 & \frac{114}{470,5} & \frac{587}{I=2368}
\end{array} \\
& \xi^{-}=5,4711 \\
& S_{\text {TOP }}=248.5 S_{\text {sor }}=432.8
\end{aligned}
$$

$$
\begin{aligned}
& M_{\text {max }}=170^{k} \times 22,75=3868 \mathrm{~m} \cdot \mathrm{k} \\
& f_{b}=\frac{M}{5}=\frac{3868}{248,5}=15,57 \mathrm{ks} i
\end{aligned}
$$

Comp. Sectroo:

$$
\begin{aligned}
& \frac{A}{2 \times 10} \quad I_{\text {AxA }}=\frac{1}{12}\left(10^{3}\right)(2)+\frac{1}{12}(1)^{3}(3) \\
& =166.9 \\
& A=20+3=23 \\
& r_{r}=\sqrt{\frac{T}{A}}=2.69 \\
& \frac{l}{r_{T}}=\frac{30.75}{2.69}=11.4 \\
& \sqrt{\frac{102 \times 10^{3} C_{b}}{36}}=53.2>\frac{l}{r_{F}} \\
& \therefore F_{b}=0,6 F_{y}=21.6>F_{b}
\end{aligned}
$$

Sre weros

OK $f=12.7 \mathrm{k} / \mathrm{mi}$

TOP

$$
\begin{aligned}
& W_{q}=\frac{V Q}{x I}=\frac{170(20)(14-5.421)}{2(2368)}=6.123 \mathrm{k} / \mathrm{im} \\
& \omega=\frac{6.123}{12.7}=0.482 \Rightarrow \text { use }_{1} 1 / 2 \text { fillets or hell groove weld }
\end{aligned}
$$

Botrom

$$
W_{q}=\frac{U Q}{x_{1}}=\frac{120(57)(5.471-2)}{2(2368)}=7.102 ; \omega=\frac{2.102}{12.7}=0.56 " 106000
$$

Use $10 \mathrm{ks}$. Electrode (E70xx); fro $0,3(70)(, 707)=14.8 \mathrm{k} / \mathrm{m}$

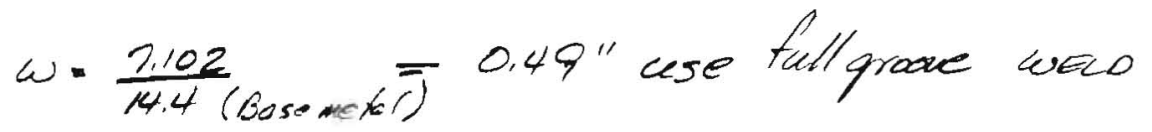



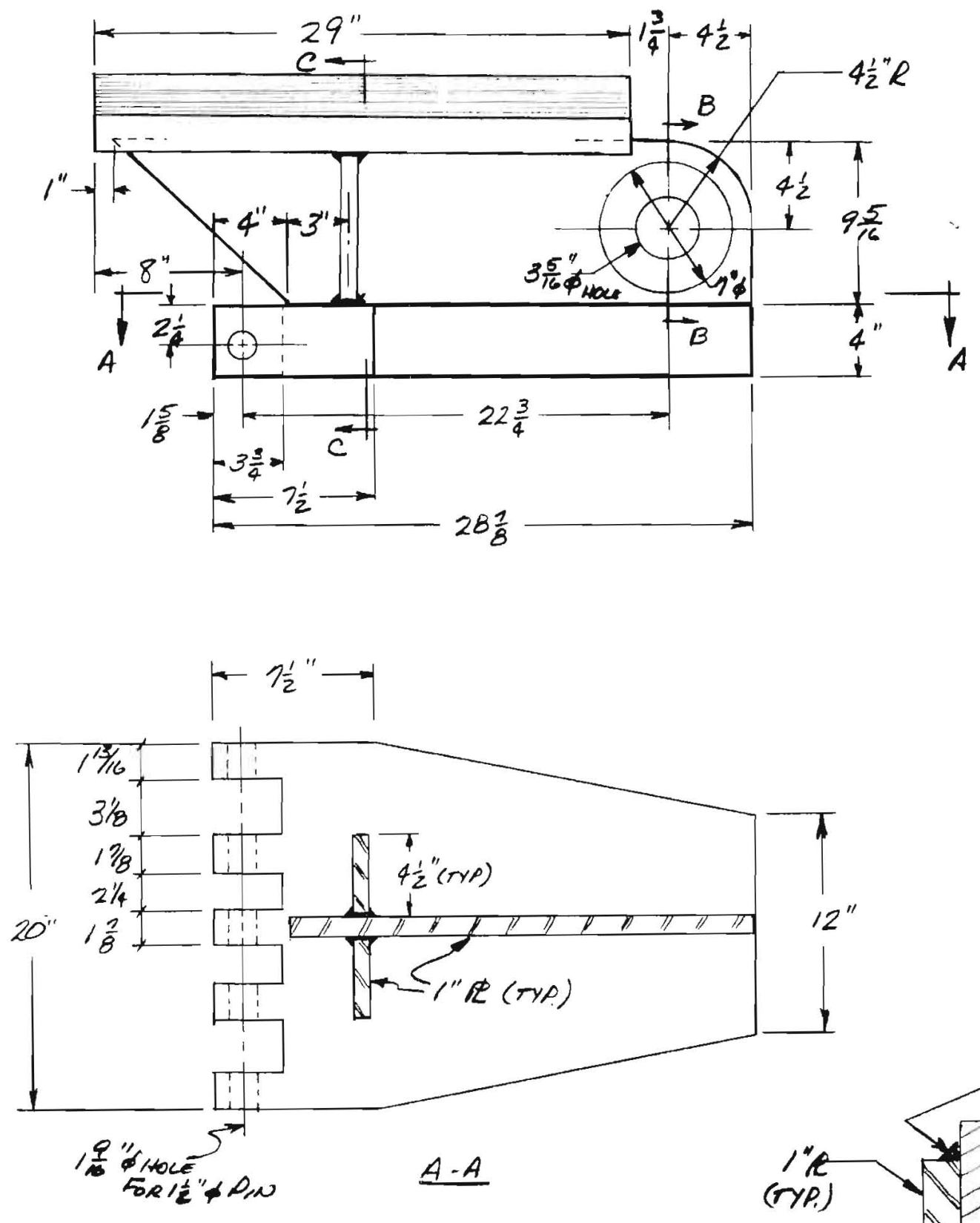

MATERIALS

PATE: A-36 OR Betrer

Pin : CAredow or LOW-AuOY

STEE HAUIAK YIELD

STEENGTH IN EXCESS

of 112 kSI.

(e.g., Stressproot).

WEIGH $\approx 900 *$

CSE CROSBY - I

\# S-2/30-85

SHACKLES

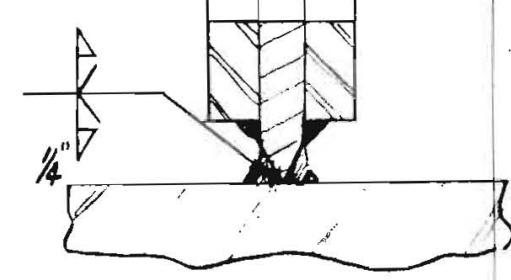


